\title{
MULTIPLICITIES, BOUNDARY POINTS, AND JOINT NUMERICAL RANGES
}

\author{
Wai-Shun Cheung, Xuhua LiU And Tin-Yau Tam
}

Abstract. The multiplicity of a point in the joint numerical range $W\left(A_{1}, A_{2}, A_{3}\right) \subseteq \mathbb{R}^{3}$ is studied for $n \times n$ Hermitian matrices $A_{1}, A_{2}, A_{3}$. The relative interior points of $W\left(A_{1}, A_{2}, A_{3}\right)$ have multiplicity greater than or equal to $n-2$. The lower bound $n-2$ is best possible. Extreme points and sharp points are studied. Similar study is given to the convex set $V(A):=\left\{x^{T} A x: x \in\right.$ $\left.\mathbb{R}^{n}, x^{T} x=1\right\} \subseteq \mathbb{C}$, where $A \in \mathbb{C}_{n \times n}$ is symmetric. Examples are given.

Mathematics subject classification (2010): Primary: 15A60.

Keywords and phrases: Joint numerical range, multiplicity, extreme point, sharp point, boundary point.

\section{REFERENCES}

[1] Y. H. AU-Yeung AND Y. T. Poon, A remark on the convexity and positive definiteness concerning Hermitian matrices, Southeast Asian Bull. Math., 3 (1979), 85-92.

[2] Y. H. Au-Yeung And N. K. Tsing, An extension of the Hausdorff-Toeplitz theorem, Proc. Amer. Math. Soc., 89 (1983), 215-218.

[3] Y. H. Au-Yeung And N. K. Tsing, Some theorems on the numerical range, Linear and Multilinear Algebra, 15 (1984), 215-218.

[4] P. L. BINDING AND C. K. LI, Joint ranges of Hermitian matrices and simultaneous diagonalization, Linear Algebra Appl., 151 (1991), 157-168.

[5] L. BRICKMAn, On the field of values of a matrix, Proc. Amer. Math. Soc., 12 (1961), 61-66.

[6] C. DAVIS, The Toeplitz-Hausdorff theorem explained, Canad. Math. Bull., 14 (1971), 245-246.

[7] W. F. Donoghue, JR., On the numerical range of a bounded operator, Michigan Math. J., 4 (1957), 261-263.

[8] M. R. EMbry, The numerical range of an operator, Pacific J. Math., 32 (1970), 647-650.

[9] R. A. Horn And C. R. Johnson, Topics in Matrix Analysis, Cambridge University Press, 1991.

[10] C. K. Li AND Y. T. Poon, Convexity of the joint numerical range, SIAM J. Matrix Anal. Appl., 21 (1999), 668-678.

[11] C. K. Li AND T. Y. TAM, Numerical ranges arising from simple Lie algebras, J. Canad. Math. Soc., 52 (2000), 141-171.

[12] A. MCIntosh, The Toeplitz-Hausdorff theorem and ellipticity conditions, Amer. Math. Monthly, 85 (1978), 475-477.

[13] Y. T. Poon, Generalized numerical ranges, joint positive definiteness and multiple eigenvalues, Proc. Amer. Math. Soc., 125 (1997), 1625-1634.

[14] J. G. STAMPFLI, Extreme points of the numerical range of hyponormal operators, Michigan Math. J., 13 (1966), 87-89.

[15] F. UHLIG, An inverse field of values problem, Inverse Problems, 24 (2008), 055019, 19 pages. 